\title{
Multi-lepton SUSY searches with the ATLAS detector
}

\author{
Katarina Pajchel - for the ATLAS collaboration
}

University of Oslo

SUSY09, Boston, June 7, 2009
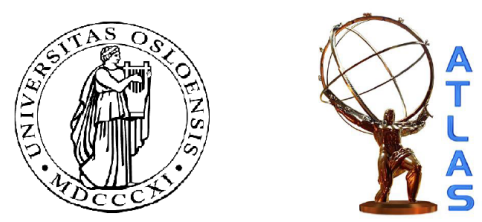


\section{Outline}

- Introduction

$\triangleright$ Why multi-lepton SUSY searches are complementary to other searches in the mSUGRA context

- Two analysis approaches - jet exclusive and jet inclusive

$\triangleright$ Analysis, results

$\triangleright$ Trigger efficiencies, systematic uncertainties

- Conclusions 


\section{Introduction}

- Minimal gravity mediated supersymmetry, mSUGRA, as example signature (R-parity conservation)

- 4 parameters: $m_{0}, m_{1 / 2}, A_{0}, \tan \beta$ and a sign

$\triangleright$ Common scalar mass $\left(m_{0}\right)$ at GUT scale: sets squark and slepton mass

$\triangleright$ Common gaugino mass $\left(m_{1 / 2}\right)$ at GUT scale: sets neutralino, chargino and gluino mass

- Translates into:

$\triangleright m_{\tilde{g}}: m_{\tilde{\chi}_{2}^{0}}: m_{\tilde{\chi}_{1}^{0}} \approx 6: 2: 1$ at Electro Weak scale

$\triangleright$ Similar quasi-fixed hierarchy in the scalar sector, e.g. $m_{\tilde{q}}>m_{i}$

- With one exception, all ATLAS benchmark points have sparticle masses below $1 \mathrm{TeV}$

Typical mSUGRA benchmark point

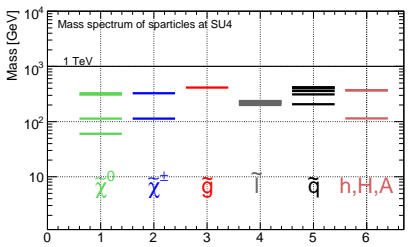

Non-typical mSUGRA benchmark point

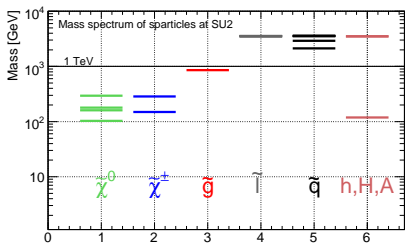




\section{Multi-lepton SUSY signal}

- Final states with 3 or more leptons from decay of heavy gauginos

$\triangleright$ If squark and gluino are light - they will dominate the SUSY cross section

$\triangleright$ Leptons from long decay chains

$\triangleright$ Hard jets

$\triangleright$ Large $\mathbb{E}_{T}$

$\triangleright$ If squarks, sleptons and possibly also

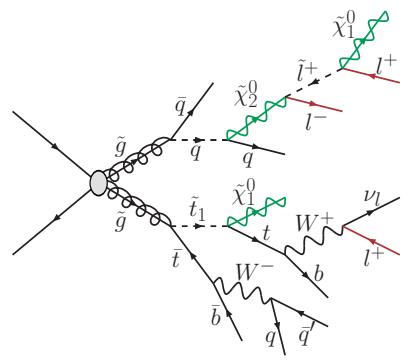
gluinos are heavy - direct gaugino production becomes important

$\triangleright$ Gauginos may decay leptonically through real or virtual $W^{ \pm}, Z^{0}$ (or sleptons, if these are not too heavy)

$\triangleright$ Low jet activity

$\triangleright$ Relatively low $\mathbb{E}_{T}$ - the gauginos are produced back to back

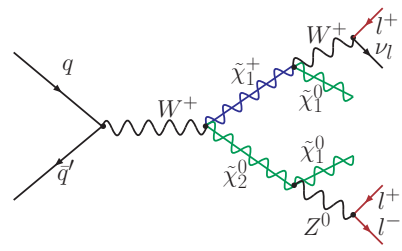




\section{Gaugino pair production}

- The so-called "Focus point region" of the SUSY parameter space (SU2) is particularly interesting

- Characterised by very heavy squarks and sleptons

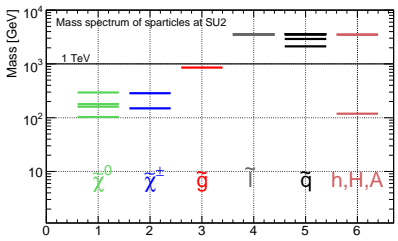

- The gauginos are light and dominate the production cross-section

- Trilepton signal mainly from the pair production of $\chi_{1}^{ \pm} \chi_{2}^{0}$

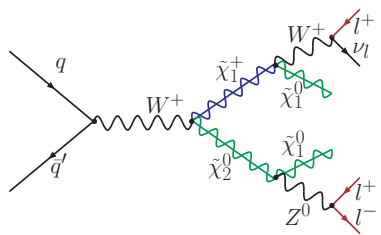

\begin{tabular}{|l|r|r|r|r|r|r|l|}
\hline Point & $m_{0}$ & $m_{1 / 2}$ & \multicolumn{1}{|c|}{$A_{0}$} & $\tan \beta$ & $\operatorname{sign} \mu$ & $\sigma_{L O}[\mathrm{pb}]$ & Point Characteristics \\
\hline SU2 & 3550 & 300 & 0 & 10 & + & 4.86 & Focus point region \\
\hline SU1 & 70 & 350 & 0 & 10 & + & 7.43 & Coanihilation region \\
\hline SU3 & 100 & 300 & -300 & 6 & + & 18.56 & Bulk region \\
\hline SU4 & 200 & 160 & -400 & 10 & + & 262.00 & Low mass point \\
\hline SU8 & 210 & 360 & 0 & 40 & + & 6.44 & Funnel region \\
\hline
\end{tabular}

- The Monte Carlo simulations are done for 14 TeV CM energy 


\section{SM Background}

- The trilepton requirement gives strong SM background suppression

The main SM backgrounds

- $t \bar{t}$ - additional lepton from semileptonic b-decay

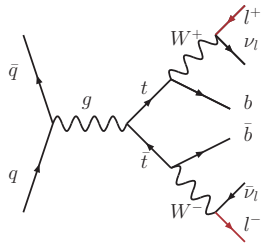

- $Z b$ - additional lepton from semileptonic b-decay

- SM counterpart: diboson production, WZ, ZZ, WW, $Z_{\gamma}$ - additional leptons from photon conversion

\begin{tabular}{|lccc|}
\hline Process & $\sigma[\mathrm{pb}]$ & k-factor & $\int \mathrm{d} t \mathscr{L}\left[f b^{-1}\right]$ \\
\hline$W W$ & 24.5 & 1.67 & 1.22 \\
$W Z$ & 7.8 & 2.05 & 2.98 \\
$Z Z$ & 2.1 & 1.88 & 12.7 \\
$Z \gamma$ & 2.6 & 1.30 & 2.98 \\
$Z b$ & 154 & 1 & 0.75 \\
$t \bar{t}$ & 450 & - & 0.92 \\
\hline
\end{tabular}
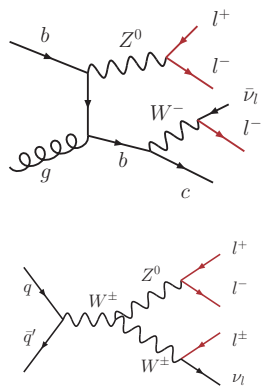


\section{Object selection}

- This analysis requires:

$\triangleright$ Efficient selection of high quality leptons

$\triangleright$ Powerful rejection of leptons from jets and leptonic $b$-quark decays

- Electrons, muons and jets

$\triangleright p_{T}>10 \mathrm{GeV}$ and within the central barrel region $|\eta|<2.5$

- Lepton isolation:

$\triangleright$ Less than $10 \mathrm{GeV}$ of transverse energy in a $\Delta R=0.2$ cone around the track

- Leptons within $\Delta R<0.4$ of a jet are rejected. $\left(\Delta R \equiv \sqrt{(\Delta \eta)^{2}+(\Delta \phi)^{2}}\right)$

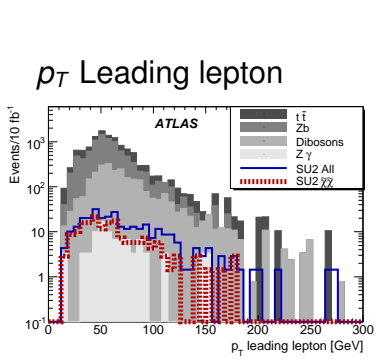

Requiring $N_{l} \geq 3$

Second leading lepton

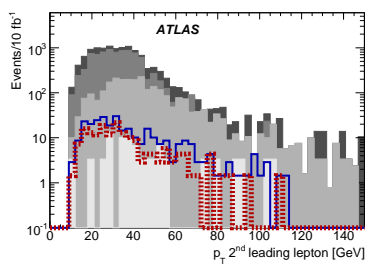

Third lepton

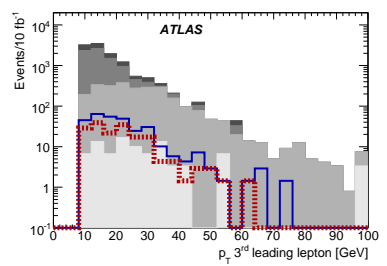




\section{Event selection}

(1) At least one pair of opposite sign, same flavour leptons (SFOS) $\left(e^{+} e^{-}\right.$or $\left.\mu^{+} \mu^{-}\right)$ with $M_{l^{++1}}>20 \mathrm{GeV}$

(2) $N_{\ell}>=3(\ell \in\{\mathrm{e}, \mu\})$

(3) $p_{T_{\text {track,max }}}^{\Delta R=0.2}<2 \mathrm{GeV}$ for electrons, $p_{T_{\text {track, } \text { max }}^{\Delta R=0}}^{\Delta R}<1 \mathrm{GeV}$ for muons, where $p_{T_{\text {track, max }}}^{\Delta R=0.2}(\ell)$ is the maximum $p_{T}$ of any track in a $\Delta R=0.2$ cone around the lepton

(4) No SFOS dilepton pair with invariant mass in the $Z^{0}$-mass window $\left|M_{S F O S}-M_{Z}\right|>10 \mathrm{GeV}$

(5) $\mathbb{E}_{T}>30 \mathrm{GeV}$ - a moderate $\mathbb{E}_{T}$ cut

(6) Optional - no jet with $p_{T}>20 \mathrm{GeV}$ - referred to as the Jet Veto 


\section{Event selection}

(1) At least one pair of opposite sign, same flavour leptons (SFOS) $\left(e^{+} e^{-}\right.$or $\left.\mu^{+} \mu^{-}\right)$ with $M_{l^{++}-}>20 \mathrm{GeV}$

(2) $N_{\ell}>=3(\ell \in\{\mathrm{e}, \mu\})$

(3) $p_{T \text { track, } \max }^{\Delta R=0.2}<\mathrm{GeV}$ for electrons, $p_{T \text { track,max }}^{\Delta R=0.2}<1 \mathrm{GeV}$ for muons, where $p_{T \text { track,max }}^{\Delta R=0.2}(\ell)$ is the maximum $p_{T}$ of any track in a $\Delta R=0.2$ cone around the lepton

(4) No SFOS dilepton pair with invariant mass in the $Z^{0}$-mass window $\left|M_{S F O S}-M_{Z}\right|>10 \mathrm{GeV}$

(5) $\mathbb{E}_{T}>30 \mathrm{GeV}$ - a moderate $\mathbb{E}_{T}$ cut

(6) Optional - no jet with $p_{T}>20 \mathrm{GeV}$ - referred to as the Jet Veto 


\section{Event selection}

(1) At least one pair of opposite sign, same flavour leptons (SFOS) $\left(e^{+} e^{-}\right.$or $\left.\mu^{+} \mu^{-}\right)$ with $M_{l^{++}-}>20 \mathrm{GeV}$

(2) $N_{\ell}>=3(\ell \in\{\mathrm{e}, \mu\})$

(3) $p_{T \text { track,max }}^{\Delta R=0.2}<2 \mathrm{GeV}$ for electrons, $p_{T \text { track,max }}^{\Delta R=0.2}<1 \mathrm{GeV}$ for muons, where $p_{T_{\text {track,max }}^{\Delta}}^{\Delta R=0.2}(\ell)$ is the maximum $p_{T}$ of any track in a $\Delta R=0.2$ cone around the lepton

(4) No SFOS dilepton pair with invariant mass in the $Z^{0}$-mass window

\section{$\left|M_{S F O S}-M_{Z}\right|>10 \mathrm{GeV}$}

\section{$\mathbb{E}_{T}>30 \mathrm{GeV}$ - a moderate $\mathbb{E}_{T}$ cut}

(6) Optional - no jet with $p_{T}>20 \mathrm{GeV}$ - referred to as the Jet Veto 


\section{Event selection}

(1) At least one pair of opposite sign, same flavour leptons (SFOS) $\left(e^{+} e^{-}\right.$or $\left.\mu^{+} \mu^{-}\right)$ with $M_{l^{+1-}}>20 \mathrm{GeV}$

(2) $N_{\ell}>=3(\ell \in\{\mathrm{e}, \mu\})$

(3) $p_{T \text { track,max }}^{\Delta R=0.2}<2 \mathrm{GeV}$ for electrons, $p_{T \text { track,max }}^{\Delta R=0.2}<1 \mathrm{GeV}$ for muons, where $p_{T \text { track,max }}^{\Delta R=0.2}(\ell)$ is the maximum $p_{T}$ of any track in a $\Delta R=0.2$ cone around the lepton

(4) No SFOS dilepton pair with invariant mass in the $Z^{0}$-mass window $\left|M_{S F O S}-M_{Z}\right|>10 \mathrm{GeV}$

(5) $\mathbb{E}_{T}>30 \mathrm{GeV}$ - a moderate $\mathbb{E}_{T}$ cut

(6) Optional - no jet with $p_{T}>20 \mathrm{GeV}$ - referred to as the Jet Veto

$M_{\text {SFOS }}$ after cut 3

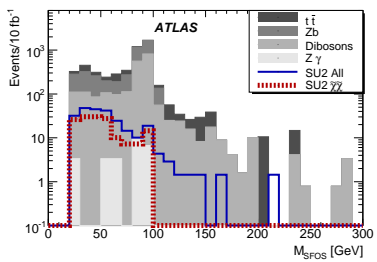




\section{Event selection}

(1) At least one pair of opposite sign, same flavour leptons (SFOS) $\left(e^{+} e^{-}\right.$or $\left.\mu^{+} \mu^{-}\right)$ with $M_{l^{+1-}}>20 \mathrm{GeV}$

(2) $N_{\ell}>=3(\ell \in\{\mathrm{e}, \mu\})$

(3) $p_{T_{\text {track,max }}}^{\Delta R=0.2}<2 \mathrm{GeV}$ for electrons, $p_{T_{\text {track,max }}}^{\Delta R=0.2}<1 \mathrm{GeV}$ for muons, where $p_{T \text { track,max }}^{\Delta R=0.2}(\ell)$ is the maximum $p_{T}$ of any track in a $\Delta R=0.2$ cone around the lepton

(4) No SFOS dilepton pair with invariant mass in the $Z^{0}$-mass window $\left|M_{S F O S}-M_{Z}\right|>10 \mathrm{GeV}$

(5) $\mathbb{E}_{T}>30 \mathrm{GeV}$ - a moderate $E_{T}$ cut

(6) Optional - no jet with $p_{T}>20 \mathrm{GeV}$ - referred to as the Jet Veto

$M_{\text {SFOS }}$ after cut 3

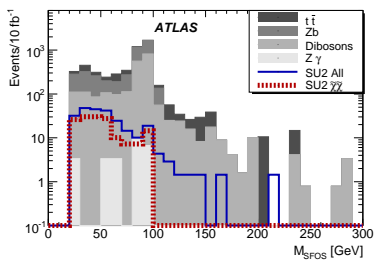

$\mathbb{E}_{T}$ after cut 4

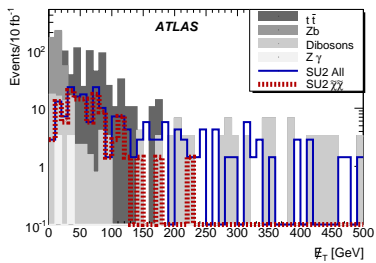




\section{Event selection}

(1) At least one pair of opposite sign, same flavour leptons (SFOS) $\left(e^{+} e^{-}\right.$or $\left.\mu^{+} \mu^{-}\right)$ with $M_{l^{+1-}}>20 \mathrm{GeV}$

(2) $N_{\ell}>=3(\ell \in\{\mathrm{e}, \mu\})$

(3) $p_{T_{\text {track,max }}}^{\Delta R=0.2}<2 \mathrm{GeV}$ for electrons, $p_{T_{\text {track,max }}}^{\Delta R=0.2}<1 \mathrm{GeV}$ for muons, where $p_{T \text { track,max }}^{\Delta R=0.2}(\ell)$ is the maximum $p_{T}$ of any track in a $\Delta R=0.2$ cone around the lepton

(4) No SFOS dilepton pair with invariant mass in the $Z^{0}$-mass window $\left|M_{S F O S}-M_{Z}\right|>10 \mathrm{GeV}$

(5) $E_{T}>30 \mathrm{GeV}$ - a moderate $E_{T}$ cut

6 Optional - no jet with $p_{T}>20 \mathrm{GeV}$ - referred to as the Jet Veto

$M_{\text {SFOS }}$ after cut 3

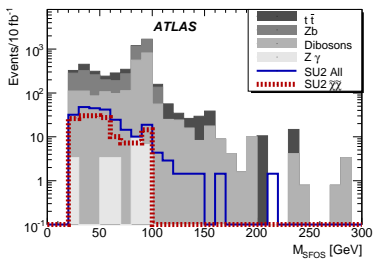

$E_{T}$ after cut 4

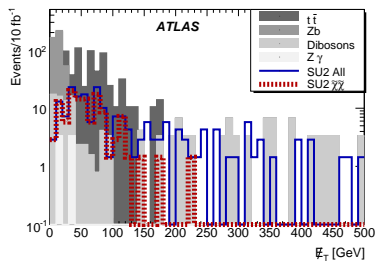

Leading jet $p_{T}$ after cut 5

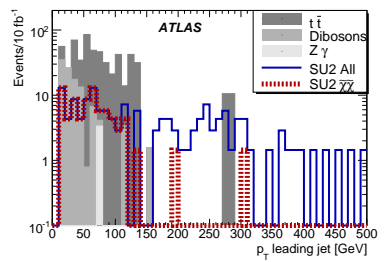




\section{The discovery potential}

- Signal significance $\mathscr{S}=\frac{S}{\sqrt{S+B}}$

$\triangleright S=$ number of signal events and $B=$ number of background events

- It has been studied for two cases:

$\triangleright$ Jet Veto - all event selection cuts, including the jet veto

$\triangleright$ Jet inclusive - all event selection cuts, except the jet veto

- The tri-lepton analysis including the jet veto with $10 \mathrm{fb}^{-1}$ :

$\triangleright 29$ Focus point region signal events (all direct gaugino), 210 SM-background events, dominated by $Z W$

$\triangleright$ Significance: $\mathscr{S}=\mathbf{1 . 9}$, which yields a $5 \sigma$ discovery after $\sim 71 \mathrm{fb}^{-1}$

- The tri-lepton jet inclusive analysis with $10 \mathrm{fb}^{-1}$ :

$\triangleright 177$ Focus point region signal events (95 of them direct gaugino), 718 SM-background events, dominated by $t \bar{t}$

$\triangleright$ Inclusive SUSY: $\mathscr{S}=5.9$

$\triangleright$ Direct gaugino: $\mathscr{S}=\mathbf{3 . 3}$ 


\section{The discovery potential - summary}

\begin{tabular}{|c|c|c|c|}
\hline & Focus p. & Focus $\chi \chi$ & Focus +JV \\
\hline $\mathscr{S}, 10 \mathrm{fb}^{-1}$ & 5.9 & 3.3 & 1.9 \\
$\int \mathrm{d} t \mathscr{L}$ for $5 \sigma$ & 7 & 23 & 71 \\
\hline & Bulk p. & Bulk $\chi \chi$ & Bulk +JV \\
\hline $\mathscr{S}, 10 \mathrm{fb}^{-1}$ & 17 & 1.9 & 1.4 \\
$\int \mathrm{d} t \mathscr{L}$ for $5 \sigma$ & 0.8 & 67 & 119 \\
\hline
\end{tabular}

\begin{tabular}{|c|c|c|c|c|}
\hline & Low mass & Bulk & Coan. & Funnel \\
\hline $\mathscr{S}, 10 \mathrm{fb}^{-1}$ & 69 & 17 & 7.7 & 1.9 \\
\hline $\mathrm{Jd} t \mathscr{L}$ ior $5 \sigma$ & & & 4.2 & \\
\hline
\end{tabular}
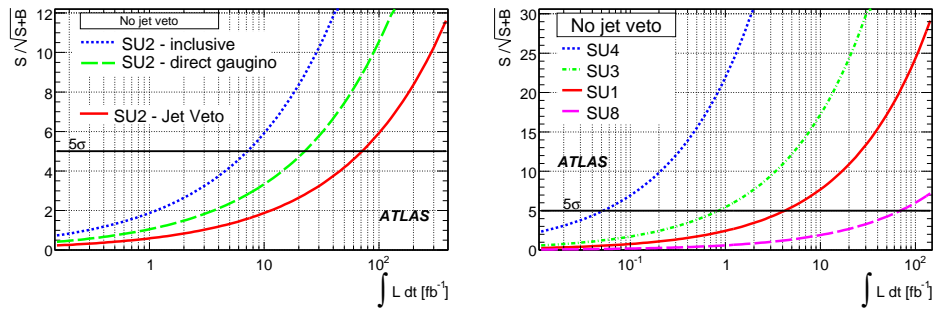

- The jet inclusive tri-lepton analysis yields a $5 \sigma$ discovery signal after less than $\sim 10 \mathrm{fb}^{-1}$ for all except one studied benchmark point.

- Including the background uncertainty (20\%), only Low Mass point (SU4) has a $5 \sigma$ discovery signal after $\sim 1 \mathrm{fb}^{-1}$ 


\section{Multi-lepton SUSY with jets, $1 \mathrm{fb}^{-1}$}

- This 3-leptons + jet analysis aims at multi-lepton production in association with jets

$\triangleright$ Cut 1: $N_{\ell}>=3$ isolated leptons (leptons with $M_{l^{+} l^{-}}<20 \mathrm{GeV}$ are discarded)

$\triangleright$ Cut 2: At least one jet with $p_{T}>200 \mathrm{GeV}$

- The effect of the single lepton triggers are included (efficiency 95\%)

- $Z_{n}>5$ for points for the Low Mass and Bulk points (SU4,SU3) even with $100 \%$ background uncertainty

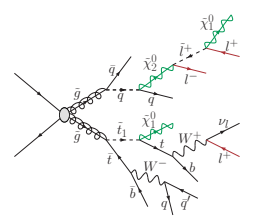

All numbers normalized to $1 \mathrm{fb}^{-1}$.

\begin{tabular}{|c|rr|rrr|}
\hline Sample & Cut 1 & Cut 2 & $S / B$ & $S / \sqrt{B}$ & $Z_{n}$ \\
\hline Focus p. & 35 & 13 & 1.1 & 3.7 & 2.7 \\
Bulk p. & 139 & 94 & 7.8 & 27.1 & 11.5 \\
Low mass & 1284 & 312 & 26.0 & 90.0 & 24.4 \\
\hline$t t$ & 455 & 11 & - & - & - \\
$Z Z$ & 59 & 0 & - & - & - \\
$Z W$ & 193 & 1 & - & - & - \\
$W W$ & 3 & 0 & - & - & - \\
$Z+\gamma$ & 9 & 0 & - & - & - \\
$Z b$ & 656 & 0 & - & - & - \\
\hline
\end{tabular}

- $Z_{n}$ - significance including the background uncertainty of $20 \%$ $Z_{n}=\sqrt{2} \operatorname{erf}^{-1}(1-2 p)$ where $p$ is the Poisson probability that the background fluctuates to the observed signal. The approximate uncertainties for $1 \mathrm{fb}^{-1}$ are estimated to be $20 \%$. 


\section{Lepton Trigger Studies - trilepton events}

- Direct gaugino production, unlike most SUSY searches:

$\triangleright$ Jet or $E_{T}$ triggers are not efficient

- Suitable triggers during early low luminosity running at $\mathscr{L}=10^{31-32} \mathrm{~cm}^{-2} \mathrm{~s}^{-1}$

$\triangleright$ Single-lepton: L2_e22i and L2_mu20

$\triangleright$ Studies at Level 2 (L2) - high probability to also pass the Event Filter

- Requiring 3 leptons with $p_{T}>10 \mathrm{GeV}$ - high probability that at least one has high $p_{T}$

- The OR combination shows good and stable performance after the $N_{l} \geq 3$ cut

\begin{tabular}{|l|ccc|ccc|ccc|}
\hline Selection & \multicolumn{3}{|c|}{ SU2 $\chi$} & \multicolumn{3}{c|}{ SU3 $\chi$} & \multicolumn{3}{c|}{ SU3 incl. } \\
Stage & L2_e22i & L2_mu20 & $\cup$ & L2_e22i & L2_mu20 & $\cup$ & L2_e22i & L2_mu20 & $\cup$ \\
\hline SFOS pair & $41 \%$ & $54 \%$ & $89 \%$ & $42 \%$ & $54 \%$ & $92 \%$ & $51 \%$ & $51 \%$ & $94 \%$ \\
SFOS+3 $^{\text {rd }} \ell$ & $58 \%$ & $67 \%$ & $93 \%$ & $59 \%$ & $63 \%$ & $95 \%$ & $66 \%$ & $68 \%$ & $98 \%$ \\
after all cuts & $57 \%$ & $66 \%$ & $92 \%$ & $58 \%$ & $57 \%$ & $94 \%$ & $66 \%$ & $64 \%$ & $97 \%$ \\
\hline
\end{tabular}

- ATLAS has three trigger levels:

$\triangle$ First level (L1) - hardware trigger

$\triangleright$ Second level (L2) - software-based

$\triangleright$ Event filter (EF) - software-based
- Trigger labels

$\triangleright$ e22i - electron, $p_{T}>22 \mathrm{GeV}$, isolated

$\triangleright$ mu20-muon, $p_{T}>20 \mathrm{GeV}$ 


\section{Systematic uncertainties - direct gaugino (Focus p.)}

- The sources of systematic uncertainties in multi-lepton search channels are different to other SUSY channels

- For $\int \mathrm{d} t \mathscr{L}=10 \mathrm{fb}^{-1}$ it is estimated to be less than $10 \%$

(1) Background production rates - large contributions from Diboson production

(2) Lepton Efficiency - based on "tag-and-probe" method

(3) Trilepton channel is very sensitive to the rate of leptons from b-decays

(4) Relevant only when using the jet veto

\begin{tabular}{|lcc|}
\hline \multirow{2}{*}{ Source } & \multicolumn{2}{c|}{ Uncertainty } \\
& No jet veto & With jet veto \\
\hline 1 Background production rates & $0.8 \%$ & $1.9 \%$ \\
2 Lepton Efficiency & $2.3 \%$ & $2.3 \%$ \\
3 Fakes $\left(R_{b \rightarrow}\right)$ ) & $4.0 \%$ & $1.2 \%$ \\
4 Hadronic energy scale & - & $1.8 \%$ \\
5 Missing energy scale & $1.5 \%$ & $1.0 \%$ \\
\hline Total systematic & $4.9 \%$ & $3.8 \%$ \\
\hline Statistical & $3.7 \%$ & $6.9 \%$ \\
\hline \hline Statistical + Systematic & $6.2 \%$ & $7.9 \%$ \\
\hline
\end{tabular}

(5) Using the jet veto - the majority of the missing energy will recoil against the leptons leading to a small missing energy scale uncertainty

- Eventually - all estimates will be done based on real data 


\section{Conclusions}

- If squarks and gluinos are heavy - dominant process: direct gaugino production

$\triangleright$ Including the jet veto $-5 \sigma$ discovery after $71 \mathrm{fb}^{-1}$

- Jet inclusive searches:

$\triangleright$ Sensitive to multi-lepton signal from a broad range of scenarios

$\triangleright$ Without the jet veto $5 \sigma$ discovery after $\sim 10 \mathrm{fb}^{-1}$ for most benchmark points

$\triangleright$ For the "Low mass point" SU4 $5 \sigma$ discovery after $\sim 1 \mathrm{fb}^{-1}$

$\triangleright$ 3-leptons + jet - strong background suppression, promising discovery potential, even with with $1 \mathrm{fb}^{-1}$

- High trigger efficiency - OR combination of lepton triggers

- Multi-lepton searches are part of the ATLAS early physics strategies 